\title{
Aggressive clinical course of extramammary Paget disease after radiotherapy
}

\author{
Yunseon Choi, MD1, Won Park, MD, PhD', Jeeyun Lee, MD, PhD², \\ Eun Yoon Cho, MD, PhD 3 , Goo-Hyun Moon, MD, PhD ${ }^{4}$ \\ Departments of ${ }^{1}$ Radiation Oncology, ${ }^{2}$ Hematology and Oncology, ${ }^{3}$ Pathology, and ${ }^{4}$ Plastic Surgery, Samsung Medical Center, \\ Sungkyunkwan University School of Medicine, Seoul, Korea
}

Extramammary Paget disease (EMPD) is a rare disease, especially in Asian populations. Surgical resection is considered the primary treatment option. Recently, radiotherapy has been suggested as an EMPD treatment, either as an alternative to surgical resection or in combination with surgical resection. This report reviewed a patient with EMPD who was treated with wide excision of the EMPD site followed by radiotherapy for remaining gross lymph node metastases. The aim of this report was to determine the optimal treatment for advanced EMPD.

Keywords: Extramammary Paget disease, Radiotherapy, Chemotherapy

\section{Introduction}

Extramammary Paget disease (EMPD) is a rare malignancy that mainly develops in the genital area [1]. The disease presents with well-defined eczematous eruption or leukokeratotic plaque, and a tendency for multifocal lesions [2]. Most cases of EMPD involve an in situ apocrine adenocarcinoma [3]. Lymph node metastasis is a poor prognostic factor for EMPD [4].

Recently, radiotherapy (RT) has been widely used to treat EMPD. RT can be used as the primary treatment in elderly patients with comorbidities who are considered medically inoperable, or in patients who want to preserve body function or structure. In addition, it can be used as an adjuvant treatment, especially in the patients who underwent incomplete resection. Patients with pathological risk factors, such as higher depth of invasion or lymphovascular invasion, also need to be treated with adjuvant RT [3].

Role of RT in the treatment of EMPD is not well-established. This report reviewed a patient with EMPD who was treated with wide excision of the primary EMPD site followed by RT for remaining gross lymph node metastases.

\section{Case Report}

A 57-year-old man with EMPD confirmed by skin biopsy came to Samsung Medical Center in September 2011. He had a slow growing scrotal plaque for the past three years (Fig. 1). On physical examination, an erythematous plaque was observed in the erythematous nodules of the left scrotum and penis (4-cm size). He did not report any itching or tenderness. Computed tomography (CT) and positron emission tomography-computed tomography (PET-CT) showed multiple

Received 26 March 2014, Revised 23 April 2014, Accepted 7 May 2014.

Correspondence: Won Park, MD, PhD, Department of Radiation Oncology, Samsung Medical Center, Sungkyunkwan University School of Medicine, 81 Irwon-ro, Gangnam-gu, Seoul 135-710, Korea. Tel: +82-2-3410-2600, Fax: +82-2-3410-2619, E-mail: wonro.park@samsung.com

(c) This is an Open Access article distributed under the terms of the Creative Commons Attribution Non-Commercial License (http://creativecommons.org/ licenses/by-nc/3.0/) which permits unrestricted non-commercial use, distribution, and reproduction in any medium, provided the original work is properly cited.

www.e-roj.org 


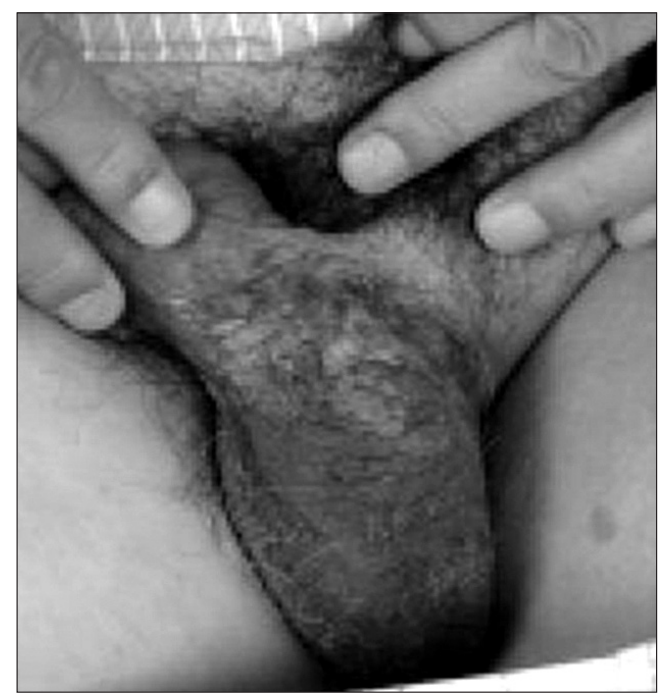

Fig. 1. Scrotal plaques associated with extramammary Paget disease.
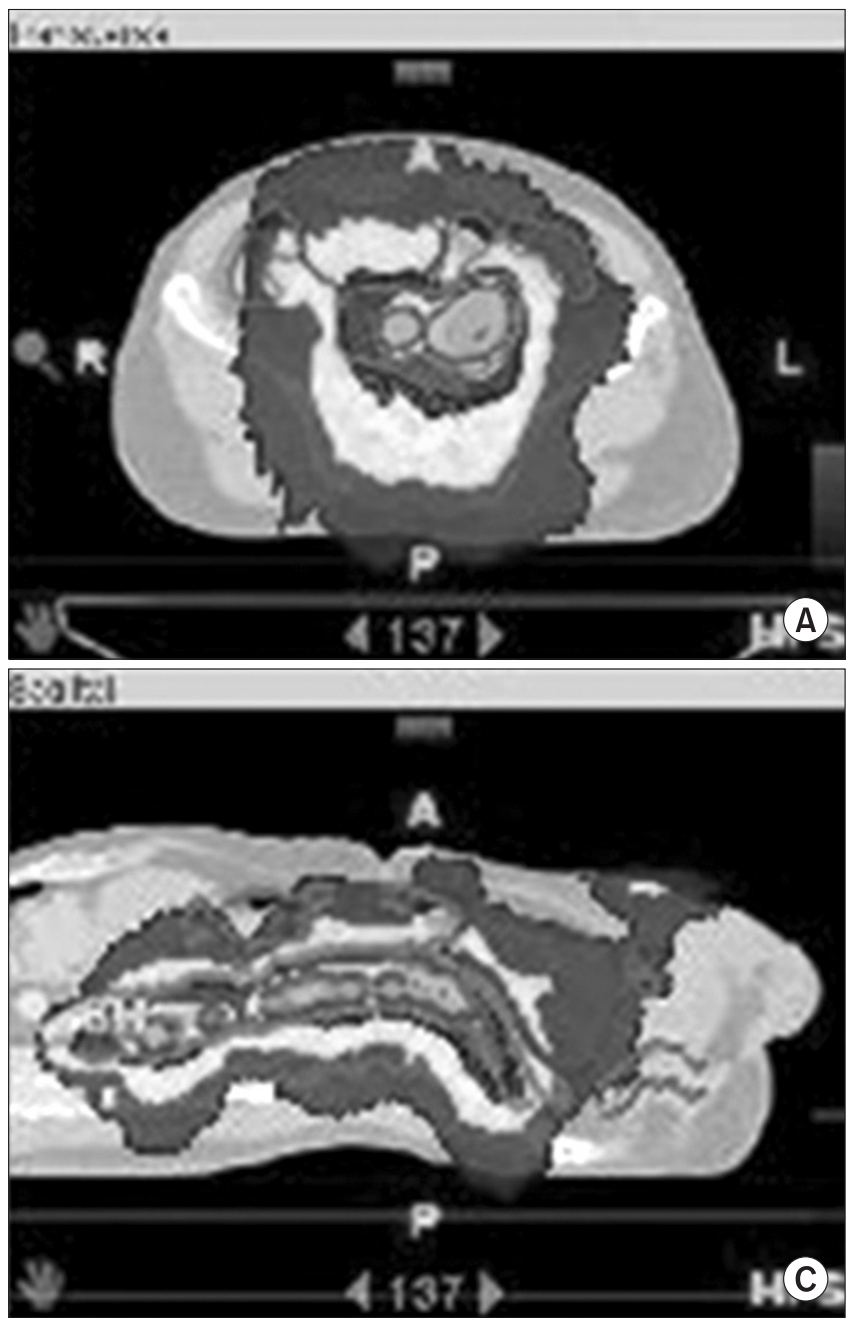

Fig. 2. Dose distributions from the intensity-modulated radiotherapy plan are shown on (A) axial, (B) coronal, and (C) sagittal view. bilateral inguinal, iliac, and retroperitoneal lymphadenopathies in preoperative evaluation. Wide excision and reconstruction with a rotation flap were performed in November 2011. According to the pathology report, the primary mass was $5 \mathrm{~cm}$ in diameter. In addition, the depth of invasion was $4 \mathrm{~mm}$, there was frequent lymphovascular invasion, and negative resection margins were reported. Proliferation of pale cells with nuclear atypia was observed on hematoxylin-eosin staining. Six weeks after surgery, the patient underwent RT to treat the bilateral inguinal pelvic and retroperitoneal lymph nodes (the primary tumor bed was excluded from the RT field) with intensitymodulated radiation therapy (IMRT) with curative intent. The RT dose for gross tumors was 62.5 Gy with 2.5 Gy per daily fraction (Fig. 2). However, in the follow-up PET-CT performed 3 months after the end of RT, newly developed hematogenous lung metastases, left supraclavicular lymph node and bilateral mediastinal lymph node metastases were found. In addition,

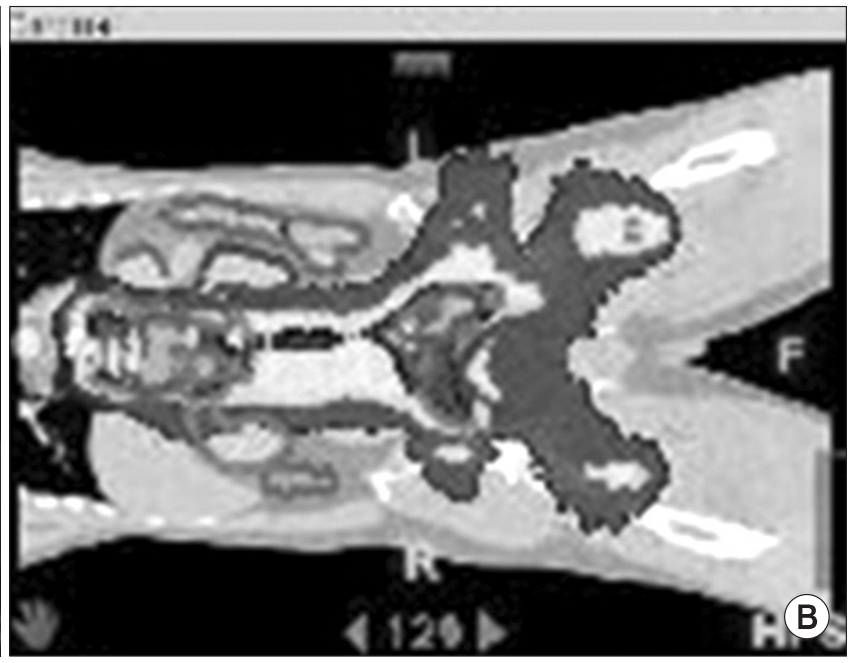



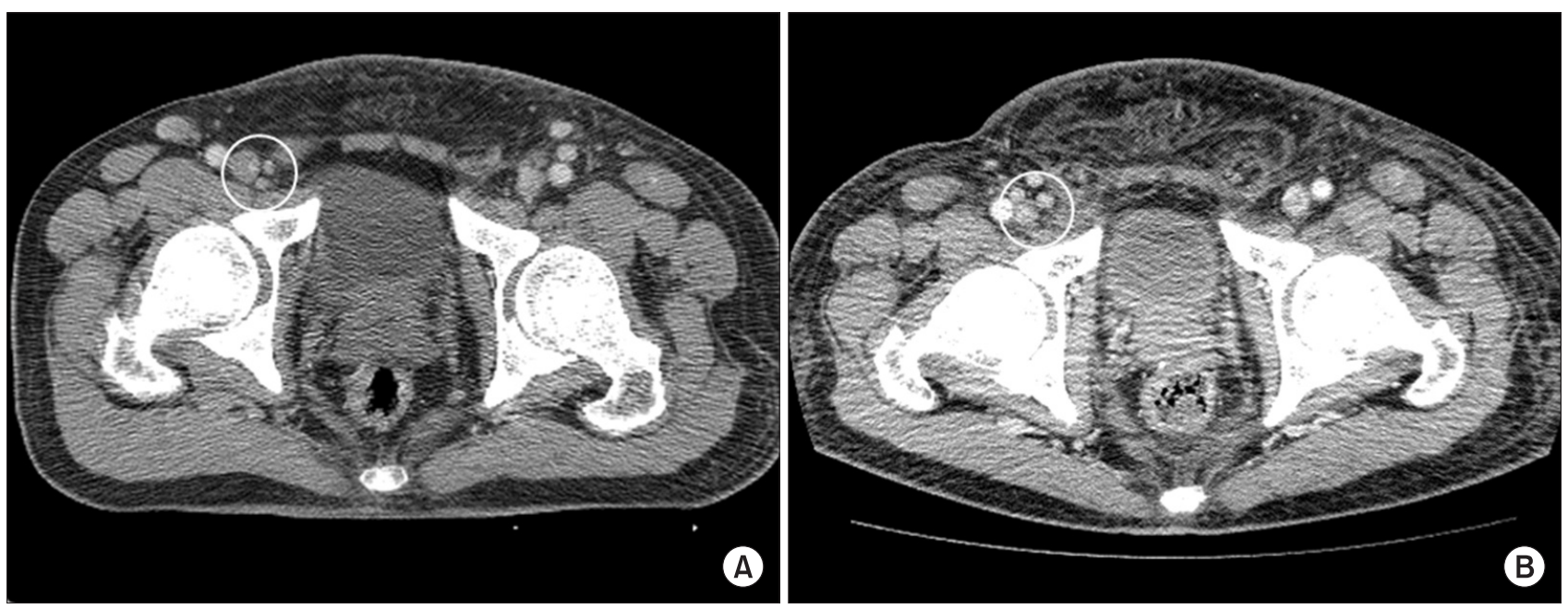

Fig. 3. Computed tomography images (A) before and (B) 13 months after radiotherapy. Growth of the right inguinal lymph nodes was noted after irradiation.

an erythematous skin lesion near the primary resection site was observed. Decreased extent of disease was observed in the previously irradiated left inguinal lymph node, aortocaval lymph node, left para-aortic lymph node, retrocrural lymph node, and left external iliac lymph node. A biopsy was performed on the left supraclavicular lymph node and scrotal skin lesion, and an EMPD relapse was confirmed. Palliative chemotherapy with etoposide and cisplatin was performed until 12 months after RT. However, disease progression in the previous RT field with growing lymph nodes along the right iliac chain and right inguinal area developed 13 months after RT (Fig. 3). In addition, multiple nodules had progressed in both lungs. The patient received second-line chemotherapy with docetaxel. Sixteen months after RT, he underwent close observation without treatment due to docetaxel-related fluid retention. Local progression with scrotal wall thickening and increased skin nodularity was observed afterwards. Seventeen months after RT, a newly developed scrotal skin lesion near the incision line was observed and biopsy confirmed recurrent invasive EMPD. Simultaneously, stable lung lesions with pleural effusion were observed in chest CT images.

\section{Discussion}

EMPD with aggressive progression after RT has been rarely reported. As for the prognostic factors for EMPD, Hata et al. [5] argued that tumor invasion into the dermis and regional lymph node metastases were significant factors for survival. Richter el al. [6] suggested that HER-2/neu overexpression was related to invasiveness of vulval EMPD. In addition, Aoyagi et al. [4] reported that combined high expression of Ki-67 was associated with invasive lesions of EMPD.

The optimal radiation dose for EMPD has not been established. In the case presented here, the patient had advanced disease at diagnosis and RT (total 62.5 Gy) for metastatic multiple lymph nodes failed to control the disease. Previous reports $[5,7,8]$ suggested that gross tumors could be treated with a total dose of $\geq 60$ Gy. However, current case showed disease progression of irradiated gross lymph nodes at 13 months after RT. Hata et al. [9] reported that gross lymph node metastases from EMPD had high rates of local control (2-year local control, 98\%) after a median total radiation of $59.4 \mathrm{~Gy}$ (range, 45 to 61.2 Gy). They argued that high dose curative RT (median radiation dose, $60 \mathrm{~Gy}$ ) for EMPD is effective in terms of local progression free survival. Luk et al. [10] also reported substantial 2-year local control rate (83\%) with equivalent total dose of 42 to 70 Gy using 2 Gy-fractions. Even though ideal dose for disease control is still unclear, radiation dose escalation in patients with gross residual disease seems to be necessary to achieve good local control. Development of RT technique (i.e., IMRT) makes it possible to deliver high radiation doses to the target while minimizing the risk of radiation-induced toxicity in surrounding normal tissue. IMRT can produce the excellent dose localization.

The patient in this case had negative surgical margins. However, he developed marginal recurrence after surgery. Though it is difficult to make any conclusion based on only this case, EMPD has a tendency to local recur even in the site 
of adequate excision. Therefore, the radiation field in adjuvant RT should be considered to cover the primary tumor site, even for the patient with negative surgical margins. Hata et al. [5] also suggested that gross tumors and postoperative surgical margins (each with a 2-cm margin) needed to be included to the clinical target volume. There is no consensus as to whether or not the RT field should include draining regional lymph nodes to block the metastatic pathway [11]. However, in cases with dermal invasion, prophylactic lymph node irradiation seems to be helpful to prevent lymph node metastases [5].

Although there are no standard chemotherapy regimens for EMPD, chemotherapy can be used to treat metastatic EMPD. Beleznay et al. [12] reported that 5-fluorouracilbased therapy with radiotherapy showed promising response. Matsushita et al. [13] recently reported a case that successfully treated metastatic EMPD with combined S-1 and docetaxel chemotherapy. Mochitomi et al. [14] reported two cases of combined chemotherapy using mitomycin, epirubicin, vincristine, cisplatin, and 5-flurouracil. In these cases, chemotherapy effectively treated metastatic lymph nodes beyond the regional lymph nodes. Molecular markers, such as HER-2 or proliferation marker Ki-67 [4,6], can be used as prognostic factors and for treatment selection. Combined chemotherapy with radiotherapy can be used for patients with systemic nodal metastases.

Proper implementation of RT is an essential part of EMPD treatment and can improve clinical outcomes. EMPD patients with systemic nodal metastases need to receive RT to primary site, even with negative surgical margins. High risk patients can be candidates for intensive treatments, such as combined chemoradiotherapy or targeted agents.

\section{Conflicts of Interest}

No potential conflict of interest relevant to this article was reported.

\section{References}

1. Siesling S, Elferink MA, van Dijck JA, Pierie JP, Blokx WA.
Epidemiology and treatment of extramammary Paget disease in the Netherlands. Eur J Surg Oncol 2007;33:951-5.

2. Lam C, Funaro D. Extramammary Paget's disease: summary of current knowledge. Dermatol Clin 2010;28:807-26.

3. Shepherd V, Davidson EJ, Davies-Humphreys J. Extramammary Paget's disease. BJOG 2005;112:273-9.

4. Aoyagi S, Akiyama M, Shimizu H. High expression of Ki-67 and cyclin D1 in invasive extramammary Paget's disease. Dermatol Sci 2008;50:177-84.

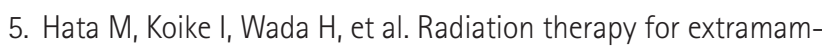
mary Paget's disease: treatment outcomes and prognostic factors. Ann Oncol 2014;25:291-7.

6. Richter $C E$, Hui $P$, Buza N, et al. HER-2/NEU overexpression in vulvar Paget disease: the Yale experience. J Clin Pathol 2010; 63:544-7.

7. Hata $\mathrm{M}$, Omura $\mathrm{M}$, Koike I, et al. Role of radiotherapy as curative treatment of extramammary Paget's disease. Int J Radiat Oncol Biol Phys 2011;80:47-54.

8. Mann J, Lavaf A, Tejwani A, Ross P, Ashamalla H. Perianal Paget disease treated definitively with radiotherapy. Curr Oncol 2012;19:e496-500.

9. Hata M, Koike I, Wada H, et al. Radiation therapy for lymph node metastasis from extramammary Paget's disease. J Eur Acad Dermatol Venereol. 2013 May 13 [Epub]. http://dx.doi. org/10.1111/jdv. 12185.

10. Luk NM, Yu KH, Yeung WK, Choi CL, Teo ML. Extramammary Paget's disease: outcome of radiotherapy with curative intent. Clin Exp Dermatol 2003;28:360-3.

11. Hatta $N$, Morita $R$, Yamada M, et al. Sentinel lymph node biopsy in patients with extramammary Paget's disease. Dermatol Surg 2004;30:1329-34.

12. Beleznay KM, Levesque MA, Gill S. Response to 5-fluorouracil in metastatic extramammary Paget disease of the scrotum presenting as pancytopenia and back pain. Curr Oncol 2009; 16:81-3.

13. Matsushita S, Yonekura K, Mera K, Kawai K, Kanekura T. Successful treatment of metastatic extramammary Paget's disease with S-1 and docetaxel combination chemotherapy. J Dermatol 2011;38:996-8.

14. Mochitomi Y, Sakamoto R, Gushi A, et al. Extramammary Paget's disease/carcinoma successfully treated with a combination chemotherapy: report of two cases. J Dermatol 2005;32:632-7. 